\title{
Missing Internal Limiting Membrane during Macular Hole Repair in Alport Syndrome
}

\author{
Sarah G. Chaudhry ${ }^{a} \quad$ Gerald Liew $^{a}, b, c, d \quad$ Adrian T. Fung ${ }^{a, b}$, c, e \\ aWestmead Hospital, Sydney, NSW, Australia; ${ }^{b}$ Westmead and Central Clinical Schools, \\ Discipline of Clinical Ophthalmology and Eye Health, Faculty of Medicine and Health, The \\ University of Sydney, Sydney, NSW, Australia; 'Westmead Institute of Medical Research, \\ Westmead, NSW, Australia; dFaculty of Medicine and Health, The University of New South \\ Wales, Sydney, NSW, Australia; e Department of Ophthalmology, Faculty of Medicine, Health \\ and Human Sciences, Macquarie University, Sydney, NSW, Australia
}

Keywords

Alport syndrome $\cdot$ Collagen IV $\cdot$ Inner limiting membrane $\cdot$ Macular hole surgery

\section{Abstract}

The aim of this manuscript is to describe a novel retinal finding of Alport syndrome during surgical management of an associated macular hole. A retrospective chart review of a 65 -yearold man with a diagnosis of Alport syndrome confirmed by renal biopsy was found to have an associated full-thickness macular hole. Pars-plana vitrectomy surgery with internal limiting membrane (ILM) peeling was attempted, but intraoperatively the ILM was found to be absent at the macula. Alport syndrome may be associated with the absence of the ILM. This can complicate attempts at macular hole repair.

\section{Introduction}

Alport syndrome is a genetic condition that affects the ocular, auditory, and renal systems [1-3]. Mutations of the COL4 gene result in dysfunctional basement membranes. In the retina, it has been known to cause parafoveal temporal retinal thinning and full-thickness macular holes. We describe a novel case of a patient with Alport syndrome who had absence of his internal limiting membrane (ILM) discovered during vitrectomy surgery for macular hole repair. 
Chaudhry et al.: Missing Inner Limiting Membrane in Alport Syndrome

\section{Case Report}

A 65-year-old emmetropic man presented with reduced vision in his left eye. He had been diagnosed with Alport syndrome at the age of 25 years by renal biopsy and had a notable family history of Alport syndrome in all of his siblings. His systemic manifestations included renal failure for which he underwent a renal transplant, sensorineural hearing loss, and systemic hypertension. Visual acuities were 20/32 right eye and 20/80 left eye. Optical coherence tomography (OCT) demonstrated marked retinal thinning bilaterally with dot-like foveal deposits in his right eye and a 453- $\mu$ m diameter full-thickness macular hole in his left eye. Alongside retinal thinning, macular holes, and fleck retinopathy, he was also noted to have posterior lenticonus and cataract, but no signs of corneal dystrophy.

The patient underwent a 25-gauge pars-plana vitrectomy for a repair of his macular hole. Intraoperatively, he was noted to have an extremely adherent posterior hyaloid, which could only be elevated to the equator, but detached without leaving residual schitic vitreous. In addition, the inner limiting membrane was found to be absent, and the retinal surface did not stain with Brilliant Blue G (ILM-Blue ${ }^{\circledR}$, DORC, Zuidland, The Netherlands) after $1 \mathrm{~min}$. Attempts to peel the ILM with ILM forceps and a Finesse Flex loop (Alcon ${ }^{\circledR}$ ), proved unsuccessful as only nerve fibre layer was present (shown in Fig. 1). This has been illustrated in the video attached to the supplementary material (shown in online suppl. Video 1; for all online

Fig. 1. a Left fundus photograph demonstrating a full-thickness macular hole with dot-like foveal deposits. b During pars-plana vitrectomy surgery, the posterior hyaloid was extremely adherent but eventually was elevated to the equator. Attempts at staining the ILM with Brilliant Blue G (ILMBLUE $®$ ) and peeling it with ILM forceps (c) and a Finesse Flex loop (Alcon ${ }^{\circledR}$ ) proved unsuccessful, as the ILM was found to be absent (d). e Preoperative OCT, demonstrating a full-thickness macular hole $453 \mu \mathrm{m}$ in diameter. There are dot-like deposits at the level of the retinal pigment epithelium within the hole, and the posterior hyaloid is still attached. It is difficult to visualize the ILM. f Postoperative OCT, showing enlargement and non-closure of the full-thickness macular hole. The posterior hyaloid is no longer visible. ILM, internal limiting membrane; OCT, optical coherence tomography.
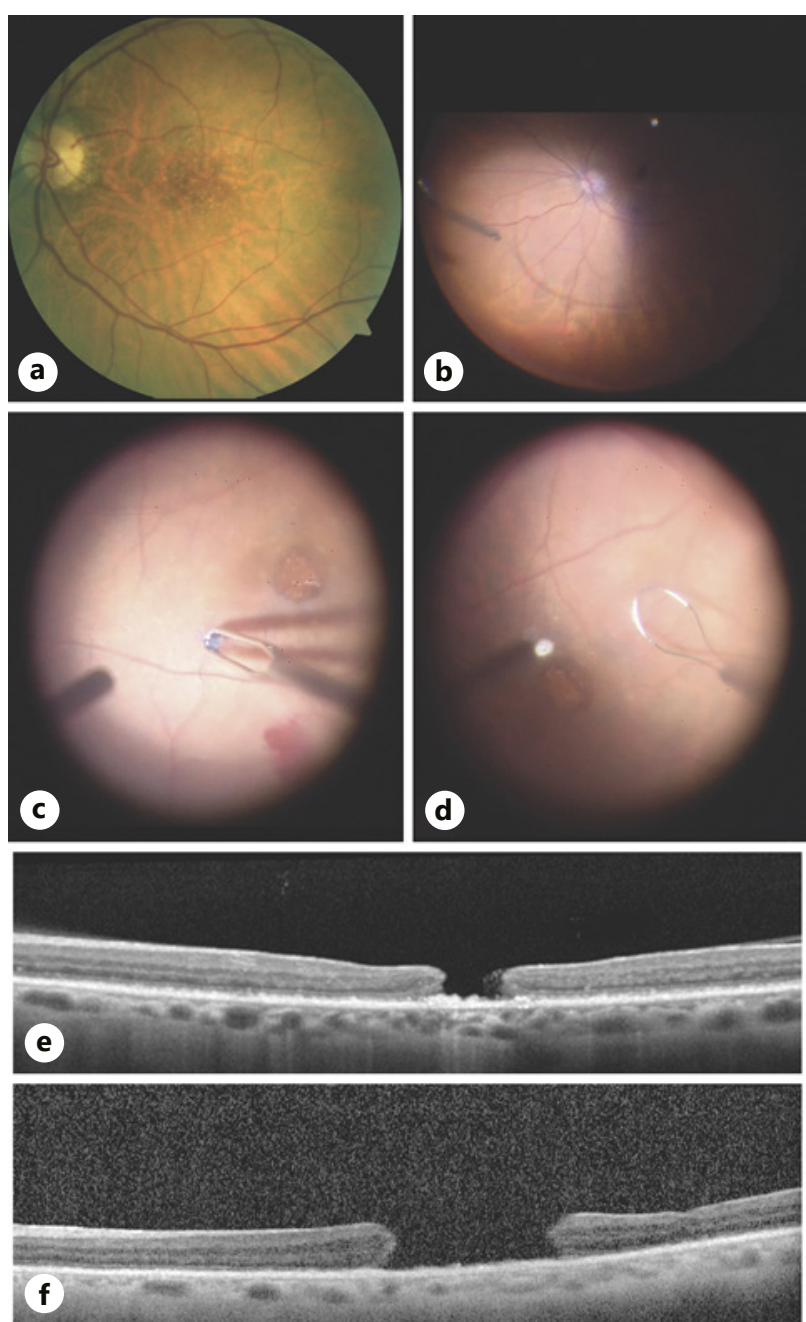
suppl. material, see www.karger.com/doi/10.1159/000513420). A fluid-gas (sulphur hexafluoride, $26 \%$ ) tamponade was used. The patient was asked to posture face down for 3 days, but despite this, the macular hole failed to close post-operatively and his visual acuity failed to improve.

\section{Discussion}

Alport syndrome is a genetic condition effecting 1 in 5,000-50,000 births [1, 2]. Inheritance is X-linked in $85 \%$ of cases, and autosomal dominant or autosomal recessive in the remainder. It primarily affects the basement membranes via mutations in the COL4 genes encoding for type-IV collagen [2]. Specifically, it has been noted that the a3 and a5 chains of type-IV collagen are most effected [2,3]. Ocular features in these patients include recurrent erosion syndrome, posterior polymorphous corneal dystrophy, anterior and posterior lenticonus, microspherophakia, anterior polar cataract, a dull macular reflex ("lozenge sign"), central and peri-macular fleck retinopathy, temporal retinal thinning ("stair-case retina"), peripheral retinoschisis, choroidal thinning, choriocapillaris flow voids, and macular holes [2-5].

Macular holes in Alport syndrome are often giant, occur without a posterior vitreous detachment and a more adherent posterior hyaloid, and are frequently present in younger patients than those with idiopathic macular holes [3,5-7]. OCT findings in these patients reveal no vitreomacular traction, reorganization around the vascular plexi of the holes and an absent foveal avascular zone. On review of the literature radially disposed cystoid macular oedema has been noted on OCT in these patients [5-8]. This is in contrast to idiopathic macular holes which appear to develop from vitreous traction with apparent posterior vitreous detachments and operculum's causing cystoid oedema, and thinning of the photoreceptor layers $[9,10]$. Hypotheses regarding the aetiology of macular holes in Alport syndrome focus on the alteration of retinal basement membranes [6]. Abnormal type-IV collagen affects the vitreoretinal interface [11]. The ILM, composed of mostly a3 and a5 type-IV collagen, has been shown to be thin on OCT scans [2]. Coalescent retinal microcystic cavities enable passage of fluid through an impaired Bruch's membrane [4,11, 12].

In our case of Alport syndrome, there was an extremely adherent posterior hyaloid and absent ILM. A MEDLINE and Pubmed search performed using the terms "Alport's AND Vitrectomy OR ILM OR Macular Hole Surgery" was used to identify other cases of macular hole surgery in patients with Alport's syndrome. Four cases were identified describing operative intervention for macular holes, with 2 describing macular hole closure [3, 6, 8, 12]. No cases described absence of the ILM.

Miller et al. [3] describe multiple failed vigorous attempts at removing the posterior cortical vitreous in a patient with Alport syndrome, such that ILM peeling was aborted. Despite this, the reported patient was noted to have closure of their macular hole 1 year after surgical intervention [3]. Randhawa et al. [8] also found elevation of the posterior hyaloid difficult but was able to eventually achieve this with successful peeling of the ILM in a 30-year-old Alport's patient resulting in a macular hole closure. Gupta and Kumar [12] were able to elevate the posterior hyaloid but did not attempt ILM peeling and did not state whether macular hole closure was achieved. Raimundo et al. $[6,12]$ describe a patient who underwent unsuccessful vitrectomy and macular hole closure at another institution.

Although thinning of the ILM has been documented on OCT scans in Alport's syndrome, this is the first cases we are aware of where ILM was found to be entirely absent on attempted macular hole repair. Our case also highlights the increased adherence of the posterior hyaloid to the retina, likely due to changes in type-IV collagen at the vitreoretinal interface. Our case

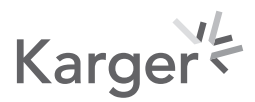


report is limited by the absence of histopathological confirmation. Nevertheless, our findings are important for advising patient preoperatively on the poorer prognosis of closing macular hole secondary to Alport syndrome than those which are idiopathic and prepare the surgeon for more challenging surgery.

\section{Statement of Ethics}

This study abided by all ethical principles in the Declaration of Helsinki involving Human Subjects. Written informed consent was obtained from the patient for publication of this case report and any accompanying images.

\section{Conflict of Interest Statement}

The authors have no conflicts of interest to disclose.

\section{Funding Sources}

The authors did not receive any funding.

\section{Author Contributions}

The first author is also the primary author for this report, and the second author is responsible for finding the case as well as the supervisor and editor.

\section{References}

1 Levy M, Feingold J. Estimating prevalence in single-gene kidney diseases progressing to renal failure. Kidney Int. 2000;58(3):925-43.

2 Savige J, Liu J, DeBuc DC, Handa JT, Hageman GS, Wang YY, et al. Retinal basement membrane abnormalities and the retinopathy of Alport syndrome. Invest Ophthalmol Vis Sci. 2010;51(3):1621-7.

3 Miller JJ, Rodriguez FJ, Smiddy WE, Rodriguez A. Macular hole surgery in Alport syndrome. Retin Cases Brief Rep. 2007;1(3):153-5.

4 Navarro R, Casaroli-Marano R, Mateo C, Gris O, Adan A, Corcóstegui B. Optical coherence tomography findings in Alport syndrome. Retin Cases Brief Rep. 2008;2(1):47-9.

5 Savige J, Sheth S, Leys A, Nicholson A, Mack HG, Colville D. Ocular features in Alport syndrome: pathogenesis and clinical significance. Clin J Am Soc Nephrol. 2015;10(4):703-9.

6 Raimundo M, Fonseca C, Silva R, Figueira J. Bilateral giant macular holes: a rare manifestation of Alport syndrome. Eur J Ophthalmol. 2019;29(1):NP13-6.

7 Shah SN, Weinberg DV. Giant macular hole in Alport syndrome. Ophthalmic Genet. 2010;31(2):94-7.

8 Randhawa S, Fu AD, Lujan BJ, McDonald HR, Jumper JM. Autofluorescence and spectral domain OCT findings in Alport syndrome. Retin Cases Brief Rep. 2013;7(4):376-9.

9 Sjaarda RN, Thompson JT. Chapter 148: macular hole. In: Ryan SJ, Hinton DR, Schachat AP, Wilkinson CP, editors. Retina. 4th ed. Edinburgh, TX: Mosby; 2006. p. 2527-44.

10 Huang J, Liu X, Wu Z, Sadda S. Comparison of full-thickness traumatic macular holes and idiopathic macular holes by optical coherence tomography. Graefes Arch Clin Exp Ophthalmol. 2010;248(8):1071-5.

11 Shaikh S, Garretson B, Williams GA. Vitreoretinal degeneration complicated by retinal detachment in alport syndrome. Retina. 2003;23(1):119-20.

12 Gupta V, Kumar N. Bilateral macular holes: an unusual feature of Alport syndrome. Retina. 2002;22(4):499501. 